\title{
Complete Anterior Capsule Phimosis following Cataract Surgery in a Patient with a History of Retinopathy of Prematurity, Nystagmus, and a Narrow Angle
}

\author{
William K. Wong Jr. ${ }^{a, b} \quad$ Malcolm R. Ing ${ }^{a, b} \quad$ Carlthan J.M. Ling ${ }^{c}$ \\ aDivision of Ophthalmology, Department of Surgery, John A. Burns School of Medicine, \\ University of Hawaii, Honolulu, HI, USA; ${ }^{b}$ Hawaii Vision Clinic, Honolulu, HI, USA; ${ }^{C} J o h n$ A. \\ Burns School of Medicine, University of Hawaii, Honolulu, HI, USA
}

\section{Keywords}

Anterior capsule phimosis - Complete occlusion of the capsular opening · Capsular contraction syndrome $\cdot$ Occluded pupil - Retinopathy of prematurity

\section{Abstract}

The authors present a case of complete anterior capsule phimosis and vision decline which developed 4 weeks postoperatively in the right eye after uncomplicated cataract surgery. Prior ocular history included retinopathy of prematurity in both eyes, acute angle closure glaucoma in the left eye, prophylactic laser peripheral iridotomy for a narrow angle in the right eye, and nystagmus in both eyes. This condition was addressed by surgically releasing the anterior capsule with microscissors to open the pupillary space which had been completely obscured by the anterior capsule, also causing the haptics of the 1-piece intraocular lens to deform. When cataract surgery was performed on his left eye, the surgeon performed prophylactic relaxing incisions at 4 points on the capsular opening. It is notable that his left eye did not develop anterior capsule phimosis postoperatively. 


\section{Introduction}

Continuous curvilinear capsulorhexis (CCC) is a common technique used for cataract surgery to provide an opening for phacoemulsification and the introduction of an intraocular lens (IOL) through the capsulorhexis opening and into the capsular bag [1]. Anterior capsule phimosis syndrome has been reported following cataract extraction in eyes with weak zonules in pseudoexfoliation syndrome [2] or in eyes with inflammation, including uveitis [3], retinitis pigmentosa [4], and diabetes [5].

The severity of the contraction and closure of the pupil determines the method of treatment utilizing either neodymium:YAG (Nd:YAG) laser or surgical excision. Treatment with $\mathrm{Nd}$ :YAG has been utilized in less severe cases in which the pupil was not completely closed. The authors report a case of a patient with a history of retinopathy of prematurity, nystagmus, narrow angle with prophylactic laser peripheral iridotomy, and cataracts who developed vision loss from a complete occlusion of the capsular opening 4 weeks following his cataract surgery in the right eye. Treatment required manual excision with microscissors. Subsequent cataract surgery on his left eye with relaxing incisions in the capsulorhexis did not result in phimosis in this eye when followed for 1 year.

\section{Case Report}

We report a case of a 40-year-old male with a history of retinopathy of prematurity, esotropia, and nystagmus. He was diagnosed in the newborn nursery with retrolental fibroplasia with a scarred retina in both eyes. At 1 year of age, it was noted that he had esotropia, nystagmus, and eccentric fixation in both eyes. He was followed yearly with routine eye exams. At the age of 4 years, the patient was noted to have myopia: right eye (OD), $-3.00 /-2.00 \times 180$; left eye (OS), $-3.00 /-2.00 \times 180$ with a visual acuity of 20/200 in both eyes (OU). His vision was stable for 30 years until he developed narrow angle closure glaucoma OS. Upon gonioscopy examination, his anterior chamber angles were determined to be grades $0-1$ OU. He was treated with i.v. mannitol, Diamox, and topical pilocarpine with subsequent argon-YAG laser iridotomy OS. He underwent prophylactic argon-YAG iridotomy OD a few weeks later for a narrow angle, which was repeated 1 week later because the original iridotomy had closed.

The patient did not develop any new ocular problems until 4 years later, when he developed recurrence of acute narrow angle closure glaucoma OS requiring a repeat YAG laser iridotomy. One year later, the patient noticed blurry vision at 20/400 OD and counting fingers OS and was diagnosed with bilateral nuclear sclerotic cataracts 3+ OD and 1-2+ OS. Two months later, an additional laser iridotomy OD was performed to open the angle before cataract surgery. The patient's axial length and anterior chamber depth were measured at 23.5 and $1.89 \mathrm{~mm}$, respectively, OD. Several weeks later, the patient was treated by an uncomplicated cataract extraction OD under general anesthesia with preoperative i.v. mannitol immediately to decrease posterior pressure and deepen the anterior chamber during surgery. A 5mm CCC was performed followed by phacoemulsification and implantation of a 1-piece aspheric hydrophobic acrylic IOL with a 6-mm optic, 13 -mm overall length, and +19.5 diopters. By the end of postoperative week 1 , the patient had restored his visual acuity back to 20/200 OD. The first 3 postoperative weeks were uneventful, but during postoperative week 4 , the patient complained of significantly decreased vision OD. His visual acuity was 20/400 OD and intraocular pressure was $7 \mathrm{~mm}$ Hg OD with Goldmann applanation. On slit-lamp examination, the patient was noted to have developed an anterior capsule phimosis with a complete 
occlusion of the capsular opening OD. The capsular contraction was so severe that the IOL haptics were folded onto the optic (Fig. 1). The option of YAG laser anterior capsulotomy was ruled out because of the patient's nystagmus, and the surgeon recognized the need to subsequently remove the capsular remnants from the pupillary space. The cataract surgeon made a decision to surgically remove the anterior capsule which had severely obscured the pupil.

The patient underwent excision of the anterior capsule OD on postoperative week 5 following his cataract surgery. During surgery, after appropriate dilation, the previous cataract incisions were opened and the aqueous was replaced with viscoelastic material. An opening was made in the anterior capsule with a 27-G needle, and some of the capsule was elevated to allow the surgeon to insert a microforceps. All residual anterior capsule in front of the IOL was removed with microscissors and the haptics were released (Fig. 2). The anterior lens capsule was biopsied and sent to the Hawaii Pathologists' Laboratory for microscopic examination. At the end of the surgery, the surgeon removed the viscoelastic material and treated the wound edges with ReSure sealant to assure a watertight wound and administered Vigamox and prednisolone drops.

The anterior lens capsule biopsied specimen was fixated and stained and examined under light microscopy (Fig. 3). Histopathological sections showed the anterior capsule (stained magenta) on periodic acid-Schiff staining (Fig. 3a) surrounded by cellular material (nuclei stained dark blue) with extracellular collagen (stained blue) on Masson's trichrome staining (Fig. 3d). Immunohistochemical examination was diffusely positive for smooth muscle actin (Fig. 3b) and focally positive for cytokeratin AE1/AE3 (Fig. 3c).

On postoperative day 1 following the capsulotomy, the visual acuity OD was now restored to $20 / 200$, which was the level at which it had been in the first week following the initial cataract surgery. The patient was treated with a topical postoperative regimen of Vigamox for 3 weeks and prednisolone acetate and Prolensa for 6 weeks. The patient's right eye had no further complication when followed for 18 months. One and a half years following the surgical removal of the anterior capsule, the patient received cataract extraction OS under general anesthesia for an increasingly dense cataract. His visual acuity OS prior to surgery was counting fingers and his axial length and anterior chamber depth were measured at 25.37 and $1.77 \mathrm{~mm}$, respectively, OS. Similar to the surgery on the right eye, a 5-mm CCC, phacoemulsification, and 1-piece aspheric hydrophobic acrylic IOL implantation with a 6-mm optic, 13-mm overall length, and +26.0 diopter were performed. However, in contrast to surgery for the right eye, the surgeon treated the left eye with 4 relaxing incisions with a Cystotome after the insertion of the IOL, each $90^{\circ}$ apart, in the capsulorhexis with the hopes of preventing the anterior capsule phimosis that had developed after surgery for the right eye. The patient's visual acuity remained at counting fingers after the surgery. It is notable that anterior capsule phimosis did not develop in his left eye when followed for 1 year.

\section{Discussion}

Anterior capsule contraction syndrome is thought to result from metaplastic lens epithelial cells (LECs) which undergo differentiation into fiber-like cells causing constriction and obstruction of the visual axis [6]. A report analyzed the capsular phimosis membrane which showed the composition of fibrous tissue interspersed with cells that resembled fibrocytes with elongated nuclei representing metaplasia of LECs [7]. Another report on a patient with anterior capsular phimosis with complete occlusion of the capsulorhexis opening showed the extracted occluding membrane to stain positive with cytokeratin (AE1-3) and SMA [8]. Our 
analysis further supports the proliferation and metaplasia of LECs into myofibroblasts as we found cellular components within the capsule specimen that were SMA and cytokeratin positive.

Inflammation and weak zonules have been found to play a role in the development of the fibrosis and contraction. The development of angle closure glaucoma in this patient possibly demonstrates a weakness of the zonules and subsequent forward displacement of the lens. The reduction of the capsulotomy opening with malposition of the opening and posterior dislocation of the IOL can cause visual impairment. Furthermore, complete anterior capsule phimosis is a rare complication of CCC, and there are only a few reports published including patients with uncomplicated cataract surgery [7, 8], retained silicone oil [9], and pseudoexfoliation syndrome [10]. To the authors' knowledge, this is the first case report of an anterior capsule contraction causing a complete obstruction of the capsular opening in a patient with a history of retinopathy of prematurity and the use of prophylactic relaxing incisions during cataract extraction to prevent capsular phimosis in the other eye.

The treatment of anterior capsule phimosis depends on the severity and progression of the contraction. In less severe cases, Nd:YAG laser anterior capsulotomy is preferred. Patients treated with Nd:YAG laser anterior capsulotomy had stable or improved vision, a capsular opening greater than the opening prior to treatment, and had no recurrence in 2 years [11]. Laser treatment has been shown to prevent the progression of the contraction using 3 or more nicks around the capsular annulus [12]. In severe or Nd:YAG laser-resistant cases, surgery with microscissors to cut the fibrotic membrane is considered. Koizumi et al. [13] performed surgery to remove the fibrous membrane on the anterior capsule edge on 2 aphakic patients with high myopia who both developed anterior capsule contraction after phacoemulsification cataract surgery, which resulted in improved visual acuity and no recurrence. In another study on 3 pseudophakic eyes and 1 aphakic eye, the membrane was removed, restoring the capsular bag to the initial shape without a relapse within a 2-year follow-up period [14]. There is also a reported uncomplicated cataract surgery case where the patient developed complete phimosis and received Nd:YAG treatment with no recurrence for several years [15].

While Nd:YAG is the preferential standard for capsulotomies in mild capsular phimosis, our case presented with multiple challenges that warranted an alternative method. One of the challenges with Nd:YAG laser capsulotomy on our patient was the presence of constant nystagmus which prevented a stable view of the anterior capsule. General anesthesia had to be utilized to immobilize the eye during surgery to provide a steady view of the capsule. Surgery with microscissors was also preferred in our case since there was a complete obstruction of the central pupillary opening. The surgeon felt that the use of the Nd:YAG laser would have resulted in large capsular fragments that would remain in the anterior chamber. Furthermore, the anterior capsule phimosis and contraction of the capsular bag had caused the haptics to deform centrally and obstruct the visual axis, requiring release and repositioning of the haptics. The patient tolerated surgery well with general anesthesia and recovered with complete clearing of the visual axis, which had been established prior to the development of anterior capsule phimosis following the initial cataract surgery. The patient did not develop anterior capsule phimosis in his left eye in which the surgeon performed prophylactic incisions on the capsular opening.

Complete anterior capsule phimosis is a rare complication of CCC. The authors report the surgical treatment of this syndrome in a patient with a history of retinopathy of prematurity. A combination of capsular peeling and excision of the anterior capsule proved to be an effective technique for remedy of severe capsular phimosis following cataract surgery. 
Prophylactic relaxing incisions during cataract surgery on the left eye may have prevented a similar complication that occurred in the right eye.

\section{Acknowledgements}

The authors would like to acknowledge the Hawaii Pathologists' Laboratory, LLP, and Dr. Tauchi-Nishi for assisting with the histopathological processing of the specimen.

\section{Statement of Ethics}

The patient provided an informed consent and permission to have this case published.

\section{Disclosure Statement}

None of the authors have any conflicts of interest.

\section{Funding Sources}

Not applicable.

\section{Author Contributions}

W.K. Wong Jr. participated with the design and concept of the study as well as being a major contributor to the revision of the manuscript. M.R. Ing contributed with revisions of the manuscript. C.J.M. Ling researched the background data on anterior capsule phimosis and drafted the manuscript. All authors approved of the final draft.

\section{References}

1 Gimbel HV, DeBroff BM. Intraocular lens optic capture. J Cataract Refract Surg. 2004 Jan;30(1):200-6.

2 Hayashi H, Hayashi K, Nakao F, Hayashi F. Anterior capsule contraction and intraocular lens dislocation in eyes with pseudoexfoliation syndrome. Br J Ophthalmol. 1998 Dec;82(12):1429-32.

3 Lüke C, Dietlein TS, Jacobi PC, Konen W, Krieglstein GK. Massive anterior capsule shrinkage after platehaptic silicone lens implantation in uveitis. J Cataract Refract Surg. 2001 Feb;27(2):333-6.

4 Hayashi K, Hayashi H, Matsuo K, Nakao F, Hayashi F. Anterior capsule contraction and intraocular lens dislocation after implant surgery in eyes with retinitis pigmentosa. Ophthalmology. 1998 Jul;105(7): 1239-43.

5 Hayashi H, Hayashi K, Nakao F, Hayashi F. Area reduction in the anterior capsule opening in eyes of diabetes mellitus patients. J Cataract Refract Surg. 1998 Aug;24(8):1105-10.

6 Marcantonio JM, Rakic JM, Vrensen GF, Duncan G. Lens cell populations studied in human donor capsular bags with implanted intraocular lenses. Invest Ophthalmol Vis Sci. 2000 Apr;41(5):1130-41.

7 Spang KM, Rohrbach JM, Weidle EG. Complete occlusion of the anterior capsular opening after intact capsulorhexis: clinicopathologic correlation. Am J Ophthalmol. 1999 Mar;127(3):343-5.

8 Edrich CL, Ghanchi F, Calvert R. Anterior capsular phimosis with complete occlusion of the capsulorhexis opening. Eye (Lond). 2005 Nov;19(11):1229-32. 


\section{Case Reports in Ophthalmology}

\begin{tabular}{l|l}
\hline Case Rep Ophthalmol 2019;10:274-280 \\
\hline DOI: 10.1159/000502282 & $\begin{array}{l}\text { @ 2019 The Author(s). Published by S. Karger AG, Basel } \\
\text { www.karger.com/cop }\end{array}$ \\
\hline
\end{tabular}

Wong Jr. et al:: Complete Anterior Capsule Phimosis following Cataract Surgery in a Patient with a History of Retinopathy of Prematurity, Nystagmus, and a Narrow Angle

9 Patton N, Ironside JW, Aslam TM, Bennett HG, Singh J. Complete occlusion of the anterior capsular opening by fibrocellular membrane associated with retained silicone oil: a clinicopathologic correlation. Retina. 2004 Jun;24(3):483-7.

10 Höhn S, Spraul CW. [Complete occlusion of the frontal capsule after cataract-operation in a patient with pseudoexfoliation syndrome-a case report and review of literature]. Klin Monbl Augenheilkd. 2004 Jun;221(6):495-7.

11 Ye H, Zhang J, Qian Y. Long-term follow-up of neodymium:YAG laser anterior capsulotomy for the treatment of anterior capsular phimosis. J Int Med Res. 2018 Sep;46(9):3692-7.

12 Hayashi K, Yoshida M, Nakao F, Hayashi H. Prevention of anterior capsule contraction by anterior capsule relaxing incisions with neodymium:yttrium-aluminum-garnet laser. Am J Ophthalmol. 2008 Jul;146(1): 23-30.

13 Koizumi K, Watanabe A, Koizumi N, Kinoshita S. Peeling the fibrous membrane from the anterior capsule for capsulorhexis contraction after phacoemulsification in aphakic patients. J Cataract Refract Surg. 2002 Oct;28(10):1728-32.

14 Reyntjens B, Tassignon MJ, Van Marck E. Capsular peeling in anterior capsule contraction syndrome: surgical approach and histopathological aspects. J Cataract Refract Surg. 2004 Apr;30(4):908-12.

15 Kim HD, Kim JM, Jung JJ. Complete occlusion of anterior capsulorhexis after uneventful cataract surgery, treated with YAG laser capsulotomy. BMC Ophthalmol. 2017 Dec;17(1):233.

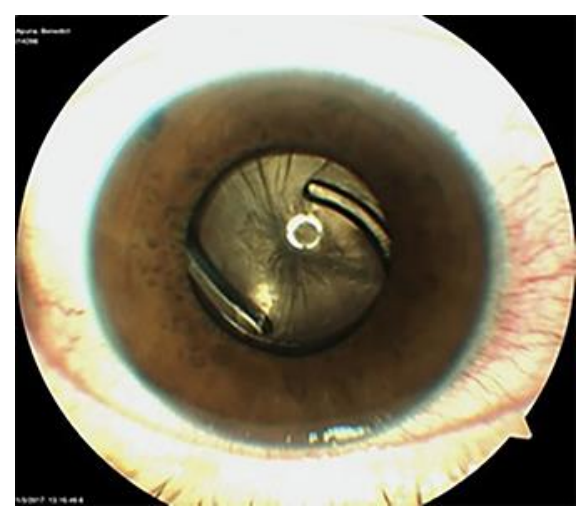

Fig. 1. Complete anterior capsule phimosis with haptics constricted in visual axis.

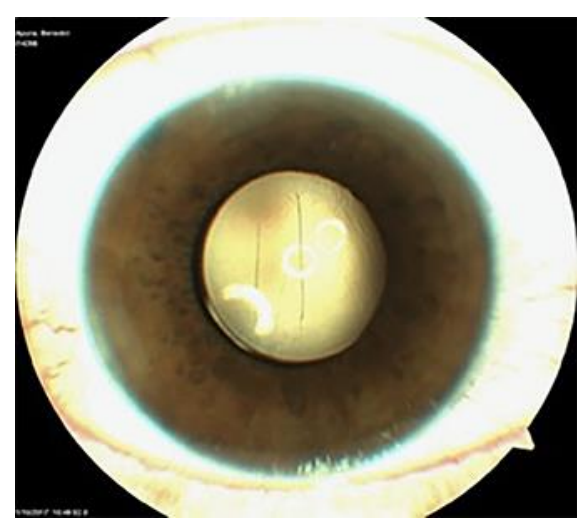

Fig. 2. Postoperative view following capsulotomy with removal of the haptics. 


\section{Case Reports in Ophthalmology}
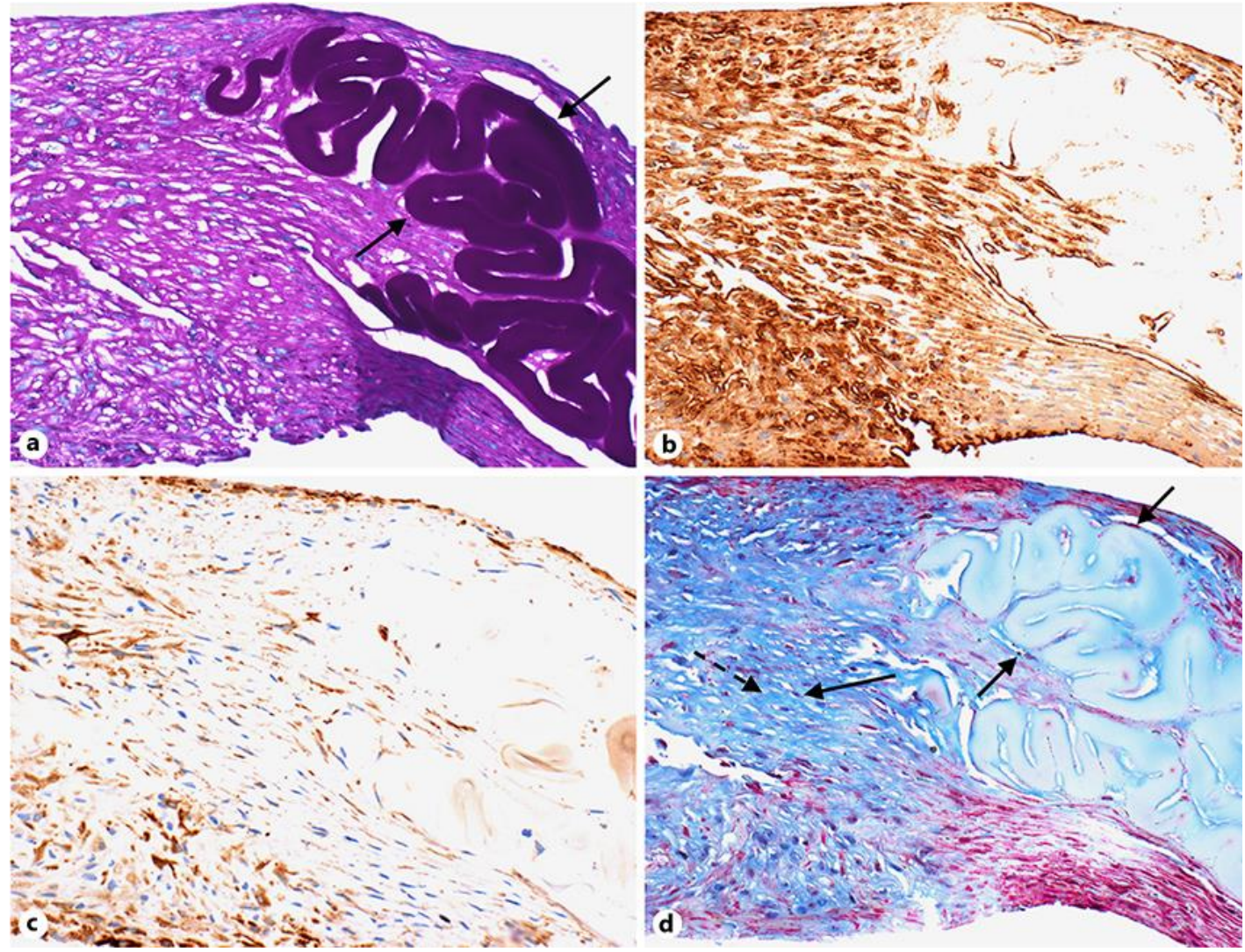

Fig. 3. Excisional biopsied specimen showed anterior capsule with proliferating metaplastic lens epithelial cells, fibroblastic changes, and contractile elements. a Histopathological section shows the anterior capsule (shown between arrows) stained magenta (periodic acid-Schiff, original magnification $\times 400$ ). Immunohistochemical staining was diffusely positive to smooth muscle actin (stained brown) (b) and focally positive to cytokeratin AE1/AE3 (stained brown) (c), both markers for myofibroblasts and epithelium (original magnification $\times 400$ ). $\mathbf{d}$ Histopathological section showed fibroblastic changes staining extracellular collagen blue (broken arrow) and nuclei dark blue (long solid arrow). The anterior capsule (short solid arrows) is again pictured on the right side of the frame and stained blue (Masson's trichrome, original magnification $\times 400$ ). 